\title{
The Characteristics of Compound Models Designed for Internet of Things
}

\author{
Jing SU, Fei MA and Bing YAO* \\ College of Mathematics and Statistics, Northwest Normal University, Lanzhou, 730070 \\ CHINA \\ *Corresponding author: yybb918@163.com
}

Keywords: Power law, 2-operator, Scale-free, Cumulative distribution, Average degree.

\begin{abstract}
In order to study the scale-free of more and more real-life networks, we design, construct a class of compound network models by the methods of graph theory for understand and try to simulate network models from Internet of Things (IoT), and we computed the parameters of models, such as: average degree, operation distribution, clustering coefficients, diameters. In the article we have verified the scale-free nature of the compound network models, that is to say, if a scale-free network $M(t)$ as base, the new network model $N(t)$ have built through the network operation on the basis of $M(t)$, it is still a scale-free network.
\end{abstract}

\section{Introduction and Concepts}

From the point of view of mathematics, it is natural to form a complex network $M(t)$ from smaller and simpler subnetworks $M_{i}(t)$ with $i=1,2, \ldots, m$. Conversely, one also hope to decompose a larger network $M(t)$ into regular and easy-handle subnetworks $M_{i}(t)$ with $i=1,2, \ldots, n$. In the above two processes, one want to keep some important properties in them. For example, if each smaller subnetwork $M_{i}(t)$ is scale-free, so is the larger network $M(t)$, and vice versa. Moreover, one want to build up high quality networks by economic methods, and maintain economically them for a long time. We will face the following problems: How to compare two networks we familiar with? By what standards to understand and characterize those networks we are interested on? By what methods to construct network models as we desired?

Doubtless, mathematics occupied an important role in researching networks. Bollobás and Riordan introduced important mathematical results on scale-free random graphs (Ref. [4]). Newman wrote over 150 articles on networks, such as: Random hypergraphs and their applications, random acyclic networks. His The structure and function of complex networks was received the most citations of any paper in mathematics between 2001 and 2011 (Ref. [5]). Newman, Barabási and Watts pointed out: "Pure graph theory is elegant and deep, but it is not especially relevant to networks arising in the real world. Applied graph theory, as its name suggests, is more concerned with real-world network problems, but its approach is oriented toward design and engineering."(Ref. [6]) Thereby, we design a class of network models, called the compound models, for trying to answer our problems in this article.

\section{A. Concepts and Definitions}

A network model in this article is a mathematical and dynamic model. A 2-operator $O_{i, j}$ of a 2-operator set $O$ is a network operation on two generators (also network models), for example, the join (see Fig.1) is a 2-operator, the cartesian product (see Fig.2) of two 
networks/graphs is a 2-operator too. Two fractal 2-operations are shown in Fig.6 and Fig.7 (Ref. [1], [2], [3],[9]). Three Sierpinski models $S(0), S(1)$ and $S(2)$ are shown in Fig.5, and furthermore $S(1)$ and $S(2)$ are constructed by the fractal 2-operation. Especially, "joining a vertex (or generator) with another vertex (or generator) by an edge" is regarded as a 2-operator on them, especially, write this 2-operator by $O o$, and the 2-operator set $O$ contains it in any time. In the real world, a 2-operation may work on the parts of networks. For example, we can take two subsets $V_{1} *=\{x, z\}$ and $V_{2}{ }^{*}=\{c, d, e, f\}$ from two models $L_{1}$ and $L_{2}$, and then do a 2-operator on them (see Fig.3), or a cartesian product (see Fig.5). Of course, the above graph operations are not deeded to appear in IoT.



Fig.1 A join product $L_{1 \vee} L_{2}$ of two models $L_{1}$ and $L_{2}$.



Fig.2 A Cartesian product $L_{1 \diamond} L_{2}$ of two models $L_{1}$ and $L_{2}$.
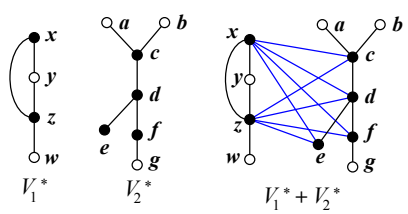

Fig.3 Two operation sets $V_{1}{ }^{*}=\{x, z\}$ and $V_{2}{ }^{*}=\{c, d, e, f\}$ from $L_{1}$ and $L_{2}$ shown in Fig. 1 produce a partial join.

For the purpose of simplicity, we require that each generator of a generator set $L(t)=\left\{L_{i}(t): 1 \leq \mathrm{i} \leq n\right\}$ to be connected (in general, it allows that each generator is connected or not), and the numbers of vertices and edges of the $j$ th generator $L_{i}(t)$ are denoted by $n_{v}^{j}(t) \geq 2$ and $n_{e}^{j}(t) \geq 1$, respectively; any two generators of $L(t)$ can be operated by a certain 2-operator $O_{i, j}$ of the 2-operator set $O$, here after. We define a type of models that are main objects in this article.

Definition 1. Suppose that $M(t)$ is a network model having $m_{v}{ }^{*}(t)$ vertices, $I(t)$ is a connected kernel of $M(t), L(t)=\left\{L_{i}(t): 1 \leq i \leq n\right\}$ is a generator set and $O=\left\{O_{i, j}: 1 \leq i, j \leq m\right\}$ is a 2-operator set with $t \in[a, b]$, defined in the above. There exists a compound network model, denoted as $C\langle M \mid I, L, O\rangle(t)$, such that: (i) each edge $x y$ of the kernel $I(t)$ corresponds to a 2-operator $O_{x, y}$ which connects two generators $L_{x}(t), L_{y}(t)$ corresponding to the ends $x, y$ of the edge $x y$; (ii) each vertex $u \in V(M(t)) \backslash V(I(t))$ that is adjacent to a vertex $v$ of $I(t)$ is joined with a certain vertex of the generator $L_{v}(t)$ corresponding to the vertex $v$.



Fig.4 Two operation sets $V_{1}^{*}=\{\mathrm{x}, \mathrm{z}\}$ and $V_{2}^{*}=\{\mathrm{c}, \mathrm{d}, \mathrm{e}, \mathrm{f}\}$ shown in Fig. 3 produce a partial cartesian product.
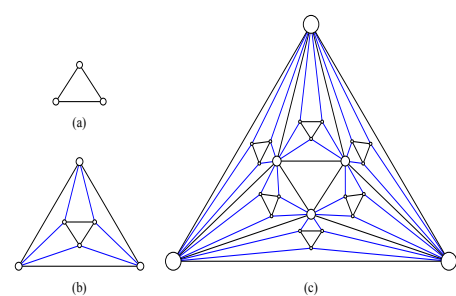

Fig.5 Three Sierpinski models: (a) $S(0)$; (b) $S(1)$; (c) $S(2)$.


Fig.6 Sierpinski model $S(1)$ is the result of a fractal 2-operation.

\section{B. Compound Models and Their Properties}

We call a compound network model $C\langle M \mid I, L, O\rangle(t)$ to be deterministic (resp. random) if $M(t)$ in Definition 1 is deterministic (resp. random). In network literature, a connected 
kernel of a network model $M(t)$, very often, is an induced graph over some specifical subset $X$ of vertex set $V(M(t))$, such as, $X$ is a dominating set, or $X$ is a set of hub vertices (having larger degrees in general), and so on. The base-model space $S(M \mid I, L, O)(t)$ contains all compound network models $C\langle M \mid I, L, O\rangle(t)$ defined in Definition 1. Vividly speaking, each $C\langle M \mid I, L, O\rangle(t)$ is a network network. The kernel $I(t)$ in the compound network model $C\langle M \mid I, L, O\rangle(t)$ varies, so does the base $M(t)$. Clearly, this evolution needs an earlier introduction for the dynamic property of the model. Let $N(\mathrm{t})=C\langle M| I, L$, $O\rangle(t)$ for the purpose of simplicity here after.

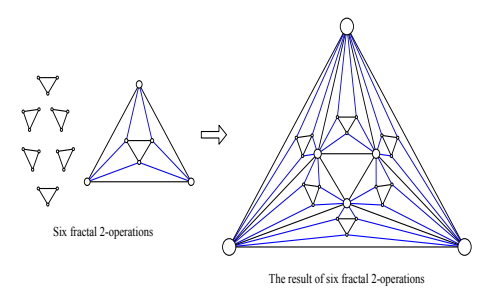

Fig.7 Sierpinski model $S(2)$ is the result of a fractal 2-operation.



Fig.8 A compound model $G^{(1)}(t)$.

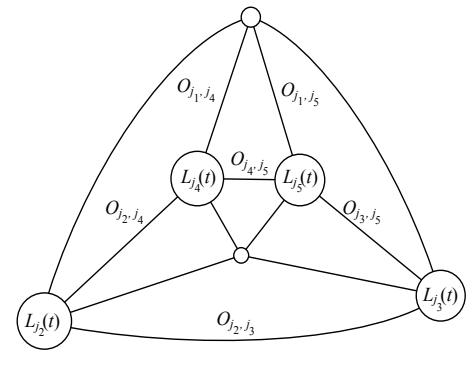

Fig.9 A compound model $G^{(2)}(t)$.

\section{Average Degrees of Compound Models}

A compound network model $N(t)$ defined in Definition 1 has its kernel $I(t)$ that is connected and not stable and quite active with $M(t)$. We may meet two situations: $I(t)=M(t)$, for the purpose of convenience, we write $N(t)$ by $G^{(1)}(t)$; or $I(t)$ is a proper sub-model of $M(t)$, we write $N(t)$ by $G^{(2)}(t)$ (see Fig.8 and Fig.9). For computation of degree spectrums of two compound models $G^{(1)}(t)$ and $G^{(2)}(t)$ at time step $t$, let $n_{v}{ }^{(s)}(t)$ and $n_{e}{ }^{(s)}(t)$ be the numbers of vertices and edges of $G^{(s)}(t)$ with $s=1,2$, respectively; $n_{v}{ }^{i}(t)$ and $n_{e}{ }^{i}(t)$ are the numbers of vertices and edges of the $i$ th generator $L_{i}(t) ; v_{i, j}{ }^{(s)}(t)$ and $e_{i, j}{ }^{(s)}(t)$ are the numbers of vertices and edges after doing a 2-operator $O_{i, j}$ on two generators $L_{i}(\mathrm{t})$ and $L_{j}(\mathrm{t})$ corresponded by two ends $x_{i}$ and $x_{j}$ of en edge $x_{i} x_{j}$ of the kernel $I(t)$ in $G^{(s)}(t)$ with $s=1,2$. By Definition 1 , we have

$$
\begin{aligned}
& n_{v}^{(s)}(t)=q_{v}^{(s)}(t)+\sum_{x_{i} x_{j} \in E(I(t))} v_{i, j}^{(s)}(t)+\sum_{i=1}^{n} c_{s, i}(t) n_{v}^{i}(t), \\
& n_{e}^{(s)}(t)=q_{e}^{(s)}(t)+\sum_{x_{i} x_{j} \in E(I(t))} e_{i, j}^{(s)}(t)+\sum_{i=1}^{n} c_{s, i}(t) n_{e}^{i}(t)
\end{aligned}
$$

with $s=1,2$, where $q_{v}^{(1)}(l)=q_{e}^{(1)}(l)=0, q_{v}^{(2)}(l)>0$ and $q_{e}^{(2)}(l)>0$ with $l=0,1,2, \ldots, t$; and $c_{s, i}(t)=0$ means that the $i$ th generator $L_{i}(t)$ of the generator set $L(t)$ does not appear in $G^{(s)}(t), c_{s, i}(t)>1$ indicates that the $j$ th generator $L_{i}(t)$ appears $c_{s, i}(t)$ times, and the vertex number $|V(I(t))|=\sum_{i=1}^{n} c_{s, i}(t)$ with $s=1,2$. Notice that $|V(I(t))|=\sum_{i=1}^{n} c_{s, i}(t)$ changes with time $t$. We define the main average degree $\overline{\langle k\rangle}_{s}^{*}(t)=\frac{2 \bar{n}_{e}^{(s)}(t)}{\overline{n_{v}(s)}(t)}$ of $G^{(\mathrm{s})}(t)$ with $s=1,2$, where $\bar{n}_{v}^{-(s)}(t)=\sum_{i=1}^{n} \frac{c_{s, i}(t) n_{v}^{i}(t)}{|V(I(t))|}, \bar{n}_{e}^{-(s)}(t)=\sum_{i=1}^{n} \frac{c_{s, i}(t) n_{e}^{i}(t)}{|V(I(t))|}$ and $\overline{\langle k\rangle_{s}}(t)=\sum_{i=1}^{n} \frac{c_{s, i}(t)}{|V(I(t))|}\langle k\rangle_{i}(t) \quad$ is the 
mean-average degree of $G^{(\mathrm{s})}(t)$ with $s=1,2$, where $\langle k\rangle_{i}(t)=\frac{2 n_{e}^{i}(t)}{n_{v}^{i}(t)}$ is the average number of $L_{i}(t)$ with $i=1,2, \ldots, n$ at time step $t$. For the first compound model $G^{(1)}(t)$, we have $I(t)=M(t)$ and $|V(I(t))|=m_{v}{ }^{*}(t)$, and

$2 \sum_{i=1}^{n} c_{1, i}(t) n_{e}^{i}(t)=\sum_{i=1}^{n}\langle k\rangle_{i}(t) c_{1, i}(t) n_{v}^{i}(t) \sim \overline{\langle k\rangle_{1}}(t) \sum_{i=1}^{n} c_{1, i}(t) n_{v}^{i}(t)$

and moreover

$$
2\left[n_{e}^{(1)}(t)-\sum_{x_{i} x_{j} \in E(M(t))} e_{i, j}^{(1)}(t)\right] \sim \overline{\langle k\rangle_{1}}(t) \sum_{i=1}^{n} c_{1, i}(t) n_{v}^{i}(t), 2\left[n_{v}^{(1)}(t)-\sum_{x_{i} x_{j} \in E(M(t))} v_{i, j}^{(1)}(t)\right]=\sum_{i=1}^{n} c_{1, i}(t) n_{v}^{i}(t),
$$

which show that the average degree $\langle\mathrm{k}\rangle^{(1)}(t)$ of $\mathrm{G}^{(1)}(\mathrm{t})$ is approximate to the mean average degree $\overline{\langle k\rangle_{1}}(t)$ for smaller values $\sum_{x_{i} x_{j} \in E(M(t))} e_{i, j}^{(1)}(t)$ and $\sum_{x_{i} x_{j} \in E(M(t))} v_{i, j}^{(1)}(t)$. Similarly with the form (2), we have $2 \bar{n}_{e}^{(1)}(t)=2 \sum_{i=1}^{n} \frac{c_{1, i}(t) n_{e}^{i}(t)}{m_{v}^{*}(t)} \sim \frac{\overline{\langle k\rangle_{1}}(t)}{m_{v}^{*}(t)} \sum_{i=1}^{n} c_{1, i}(t) n_{v}^{i}(t)$, and the main average degree

$$
\langle k\rangle_{i}^{*}(t)=\frac{2 n_{e}^{-(1)}(t)}{\bar{n}_{v}^{(1)}(t)} \sim \overline{\langle k\rangle_{1}}(t)
$$

Therefore, $G^{(1)}(t)$ has its own average degree $\frac{2 n_{e}^{(1)}(t)}{n_{v}^{(1)}(t)}=\langle k\rangle^{(1)}(t) \sim{\overline{\langle k\rangle_{1}}}^{*}(t) \sim \overline{\langle k\rangle}_{1}(t)$. The above facts give us a result

Theorem 1. The base-model space $S^{(1)}=\left\{G^{(1)}(t): M \mid I, L, O\right\}$ is closed to the sparseness if each generator $L_{j}(t)$ and $M(t)$ are sparse in $G^{(1)}(t)$.

The from (2) and the result (3) show that $G^{(1)}(t)$ is spares if every generator of the generator set $L(t)$ is spares, since the main average degree $\overline{\langle k\rangle}_{1}^{*}(t)=\frac{2 n_{e}^{(1)}(t)}{n_{v}^{(1)}(t)}$ converges to the average degree $\langle k\rangle^{(1)}(t)$ of $G^{(1)}(t)$ as $t \rightarrow \infty$ under the smaller values of $X^{(1)}(t)=\sum_{E(M(t))} v_{i, j}^{(1)}(t), Y^{(1)}(t)=\sum_{E(M(t))} e_{i, j}^{(1)}(t)$.

However, we can not say that the second compound model $G^{(2)}(t)$ to be spares, since it is not easy to show the main average degree $\overline{\langle k\rangle}_{2}^{*}(t) \sim\langle k\rangle^{(2)}(t)$ for large $t$. For dealing with various parameters of $G^{(2)}(t)$, we have to consider the values of two numbers $X^{(2)}$ and $Y^{(2)}$, where $X^{(2)}(t)=q_{v}^{(2)}(t)+\sum_{E(I(t))} v_{i, j}^{(2)}(t), Y^{(2)}(t)=q_{e}^{(2)}(t)+\sum_{E(I(t))} e_{i, j}^{(2)}(t)$, since $q_{v}^{(2)}(t)$ and $q_{e}^{(2)}(t)$ may be quite large or active extensively.

\section{Operation Distribution of $G^{(1)}(t)$}

Since the kernel $I(t)$ is equal to the base $M(t)$ in $G^{(1)}(t)$, we define: $K_{i}^{*}$ is an operation degree for which a randomly selected generator $L_{i}(t)$ having been operated with other $K_{i}^{*}$ generators in $G^{(1)}(t) ; P\left(K_{i}^{*}\right)$ is the operation distribution of $G^{(1)}(t)$, that is, the probability of a randomly selected generator $L_{i}(t)$ operated with other $K_{i}^{*}$ generators; $\Pi_{i}^{*}=K_{i}^{*}(t) / \sum_{j} K_{j}^{*}(t)$ is the probability of a new added generator $L_{j}(t)$ operated with a 
generator $L_{i}(t)$ that was already operated with other generators $K_{i}^{*}$ times. Very often, $\Pi_{i}^{*}=K_{i}^{*}(t) / \sum_{j} K_{j}^{*}(t)$ is called the linear preferential attachment (Ref. [7]).

Experiment. By means of BA-model, we add a new generator to $G^{(1)}(t-1)$ and operate it with $m$ generators of $G^{(1)}(t-1)$ by using $m$ 2-operators, so we have a dynamic operation equation (Ref. [10], [11], [12], [13])of $G^{(1)}(t)$ as

$$
\frac{\partial K_{i}^{*}(t)}{\partial t}=m \frac{K_{i}^{*}(t)}{\sum_{j} K_{j}^{*}(t)}
$$

Suppose that $G^{(1)}(0)$ has been generated by $n_{0}$ generators and $m_{0}$ operators used. At time step $t, G^{(1)}(t)$ contains $n_{0}+t$ generators and $m_{0}+m t$ operators in total. Thereby, $\sum_{j} K_{j}^{*}(t)=2\left(m_{0}+m t\right)$. Under $K_{i}^{*}\left(t_{i}\right)=m$, we can solve $K_{i}^{*}(t)=\sqrt{\frac{m_{0}+m t}{m_{0}+m t_{i}}}$, and $t_{i}>M(t, K)=\frac{m_{0}+m t}{m K^{2}}-\frac{m_{0}}{m}$ after setting $K_{i}^{*}(t)<K$. Moreover, the density function $p\left(t_{i}\right)=1 /\left(m_{0}+t_{i}\right)$ according to the well-distributed distribution enables us to compute

$$
P\left(K_{i}^{*}(t)<K\right)=P\left(t_{i}>M(t, K)\right)=1-P\left(t_{i} \leq M(t, K)\right)=1-\int_{0}^{M(t, K)} p(t) d x=1-\frac{M(t, K)}{m_{0}+t}
$$

So, $P(K)=\frac{\partial P\left(K_{i}^{*}(t)<K\right)}{\partial K} \sim \frac{m_{0}+m t}{m\left(m_{0}+t\right)} \cdot \frac{2}{K^{3}}$ as desired.

In general, we say $G^{(1)}(t)$ to be a scale-free operation model if $G^{(1)}(t)$ holds its own operation distribution $P(K) \sim K^{-\lambda}$ with $2<\lambda<3$. Such models having the scale-free operation can be found in social networks in the the real life. The above facts induce a concept as follows

Definition 2. A compound model $N(t)$ is a uniformly scale-free model if each generator $L_{i}(t)$ of the compound model holds its own degree distribution $P_{i}(k) \sim k^{-\gamma_{i}}$ with $2<\gamma_{i}<3$ and the compound model has its own operation distribution $P(K) \sim K^{-\lambda}$ with $2<\lambda<3$.

As refering "joining a vertex (or generator) with another one by an edge" as a 2-operator, so Definition 2 can be applied to each compound model defined here. Let $P(k)$ be the degree distribution of $G^{(1)}(t), P_{M(t)}(k)$ be the degree distribution of the base $M(t)$, the notation $\left|L_{j}(t)\right|_{1}^{n} \rightarrow 1$ indicates that each generator $L_{j}(t)$ contracts to a vertex. Thereby, we have a connection of three distributions of $G^{(1)}(t)$ as follows

$$
\lim _{\left|L_{j}(t)\right|_{1}^{n} \rightarrow 1} P(k)=P_{M(t)}(k)=P(K)
$$

\section{E. Clustering Coefficients of $G^{(1)}(t)$}

We define the base-clustering coefficient $\langle c\rangle_{\text {base }}^{(1)}$ of the compound model $G^{(1)}(t)$ is the clustering coefficient of the base $M(t)$ in $G^{(1)}(t)$. In other words, $\langle c\rangle_{\text {base }}^{(1)}$ is a clustering coefficient between networks, rather than between vertices in $G^{(1)}(t)$. The clustering 
coefficient $\langle c\rangle_{j}$ of the $j$ th generator $L_{j}(t)$ is called a partial clustering coefficient of $G^{(1)}(t)$, denoted as $\partial_{j}\langle c\rangle=\langle c\rangle_{j}$. So, we have the mean-clustering coefficient

$$
\overline{\langle c\rangle}\rangle^{(1)}=\sum_{j=1}^{n} \frac{c_{1, j}(t)}{m_{v}^{*}(t)} \partial_{j}\langle c\rangle=\sum_{j=1}^{n} \frac{c_{1, j}(t)}{m_{v}^{*}(t)}\langle c\rangle_{j}
$$

It can be thought that $\overline{\langle c\rangle}(1)$ is local, $\langle c\rangle_{\text {base }}^{(1)}$ is universal. We define the efficient clustering coefficient of $G^{(1)}(t)$ as follows.

$$
\langle c\rangle_{\text {base }}^{(1)} \cdot\langle\bar{c}\rangle^{(1)}=\sum_{j=1}^{n} \frac{c_{1, j}(t)}{m_{v}^{*}(t)}\langle c\rangle_{j} \cdot\langle c\rangle_{\text {base }}^{(1)}
$$

Similarly, it can define various clustering coefficients for the compound model $G^{(2)}(t)$.

\section{F. Diameters of Compound Models}

Bolloba's and Riordan [8] have proven that the diameter $\operatorname{diam}\left(G_{m}{ }^{n}\right)$ of a scale-free graph $G_{m}^{n}$ on $n$ vertices holds

$$
\frac{(1-\varepsilon) \log n}{\log \log n} \leq \operatorname{diam}\left(G_{m}^{n}\right) \leq \frac{(1+\varepsilon) \log n}{\log \log n}
$$

Some papers quote: Scale-free graphs of order $n$ have diameters no more than $c \log n / \log \log n$ (or $c \ln n / \ln \ln n$ ). Similarly with the deduction in the previous subsection, we have

Theorem 2. If $M(t)$ and all generators of $N(t)$ are small-world, then the base-model space $S(M \mid I, L, O)(t)$ is closed to the small-world property of networks.

\section{Conclusion and Problems}

The researchers rarely involved in the network operation research, in this paper we defines several network operation, and on the basis of it we have designed two types of compound models $G^{(1)}(t)$ and $G^{(2)}(t)$ from social and logistical networks. Furthermore, we discuss the properties of the compound models, such as degree distribution, diameter, average degree, clustering coefficient, and so on. Based on the particular construction of $G^{(1)}(t)$, the operation distribution of $G^{(1)}(t)$ is a new concept. In the article we have verified the scale-free nature of the compound network models, our works on network have a certain guiding role for the later research in compound network models. As further works on designing network models, we present some problems on the compound models.

Problem 1. Find some connections between the degree distribution $P(k)$ and the operation distribution $P(K)$ of a compound model.

Problem 2. If a compound model is uniformly scale-free, is it scale-free?

\section{Acknowledgement}

This research was supported by the National Natural Science Foundation of China under grants No. 61163054, No. 61363060 and No. 61662066. 


\section{References}

[1] Bing Yao, Jing Su, Fei Ma, Xiaomin Wang, Xiyang Zhao, Ming Yao. Exploring Network Operations For Data and Information Networks. submitted (2016)

[2] Bing Yao, Xiaomin Wang, Jing Su, Fei Ma, Ming Yao, Mingjun Zhang, and Jianmin Xie. Methods And Problems Attempt in Scale-Free Models From Complex Networks. submitted (2016).

[3] Bing Yao, Jing Su, Fei Ma, Xiaomin Wang, Hui Sun, Ming Yao. Network Models Made By Dynamic Differential Equations. submitted (2016)

[4] Be'la Bolloba's and Oliver M. Riordan, Handbook of Graphs and Networks From the Genome to the Internet, WILEY-VCH GmbH and Co. KGaA, Weinheim, 2003 (https://en.wikipedia.org/wiki/Bela-Bollobas).

[5] https://en.wikipedia.org/wiki/Mark-Newman

[6] M. E. J. Newman, A.-L. Barab'asi, and D. J.Watts, The Structure and Dynamics of Networks. Princeton University Press, Princeton (2006).

[7] bert-La'szlo' Baraba'si and Reka Albert. Emergence of scaling in random networks. Science 286 (1999) 509-512.

[8] B. Bolloba's and O. Riordan. The Diameter of a Scale-Free Random Graph. Combinatorica 24:1 (2004), 5-34.

[9] Zhang Zhongzhi, Zhou Shuigeng, Fang Lujun, Guan Jihong, Zhang Yichao. Maximal planar scale-free Sierpinski networks with small-world effect and power-law strength-degree correlation. EPL (Europhysics Letters), 2007, 79: 38007.

[10] Bing Yao, Xiaomin Wang, Jing Su, Fei Ma, Xiyang Zhao, Ming Yao, Mingjun Zhang and Guoxing Wang. Methods And Problems Attempt in Scale-Free Models From Complex Networks.Joint International Information Technology, Mechanical and Electronic Engineering Conference (JIMEC 2016), ISSN 2352-5401, Volume 59, pp57-61. ISBN: 978-94-6252-234-3, ATLANTIS PRESS, 2016

[11] Bing Yao, Fei Ma, Jing Su, Xiaomin Wang, Xiyang Zhao, Ming Yao. Scale-Free Multiple-Partite Models Towards Information Networks. Proceedings of 2016 IEEE Advanced Information Management, Communicates, Electronic and Automation Control Conference (IMCEC 2016) pp549-554. Compliant PDF Files: IEEE Catalog Number: CFP16E32-ART ISBN: 978-1-4673-9613-4; Conference CD-ROM Version: IEEE Catalog Number: CFP16E32-CDR ISBN: 978-1-4673-9611-0; Print Version: IEEE Catalog Number: CFP16E32-PRT ISBN: 978-1-4673-9612-7

[12] Fei Ma, Jing Su, Bing Yao, Ming Yao. Scale-free Network Models With Parameters. Joint International Information Technology, Mechanical and Electronic Engineering Conference (JIMEC 2016), ISSN 2352-5401, Volume 59, pp155-162. ISBN: 978-94-6252-234-3, ATLANTIS PRESS, 2016

[13] Jing Su, Fei Ma, Bing Yao, Ming Yao. AS-mixed Network Models Created By Triangle-expanding Operations. Joint International Information Technology, Mechanical and Electronic Engineering Conference (JIMEC 2016), ISSN 2352-5401, Volume 59, pp260-265. ISBN: 978-94-6252-234-3, ATLANTIS PRESS, 2016 